\title{
Teoría de redes y fenómenos de comunicación:
} problemas metodológicos

\author{
José Luis Plottier
}

\begin{abstract}
José Luis Plottier::
Departamento de Comunicación, Facultad de Ciencias Humanas, Universidad Católica del Uruguay, Uruguay. jlplottier@gmail.com
\end{abstract}

\section{RESUMEN}

La teoria de redes se ha desarrollado en paralelo con la difusión masiva de las redes digitales (Internet y la telefonía móvil). A través del enfoque transdisciplinario de esta teoria se observa una verdadera invasión de científicos duros en el ámbito de las ciencias sociales, en particular en el del estudio de los fenómenos de comunicación. Este proceso de endurecimiento de las ciencias sociales tiene sin duda muchos efectos positivos, pero al mismo tiempo genera problemas metodológicos. En este trabajo se describe brevemente dicho proceso y se analizan algunos de los problemas metodológicos que conlleva. Se concluye que es necesaria una convergencia metodológica entre las tradiciones de investigación de las ciencias durasy las de las ciencias sociales, para que la investigación en comunicación pueda dar el salto epistemológico que las redes digitales del siglo XXI requieren.

En este trabajo ${ }^{1}$ intento darle al lector un panorama inicial sobre algunos aspectos relevantes de la investigación cuantitativa sobre redes digitales en el marco conceptual definido por la teoría de redes (en adelante TR) y discutir algunos de los problemas metodológicos que el desarrollo de dicha teoría supone en relación con la investigación en comunicación social. Corresponde que haga dos salvedades al respecto.

La primera tiene que ver con los métodos matemáticos y estadísticos que utiliza la TR. Mis referencias a ellos van a ser breves, ceñidas a las ideas más básicas, y desprovistas de rigor teórico (con el objetivo de hacer más fácil la lectura para quien no ha tenido mayor contacto con estos temas). segunda edición de Plottier (2008), actualmente en preparación.
La segunda refiere al abordaje transdisciplinario de la TR, que tiene aplicaciones en dominios muy diferentes:

\section{ABSTRACT}

Development of network theory has advanced in parallel with the massive dissemination of digital networks (Internet and wireless telephony). Using the transdisciplinary approach of this theory, a veritable onslaught of "hard" scientists has been observed in the field of social sciences, more particularly in the study of communication phenomena. While this "hardening" process of social sciences presents, undoubtedly, many positive aspects, at the same time it generates methodological problems as well. That process is briefly described in this article, and some of the problems "hardening" entails are analyzed. The conclusion points to a necessary convergence in traditional research methodologies from both fields, "hard" sciences and social sciences, so that communication research is enabled to jump ahead epistemologically, in order to satisfy the demands of digital networks in the twenty-first century.

desde la ecología hasta la comunicación social, pasando por la epidemiología, la neurología, la economía y las redes de computadoras, para mencionar algunos. Ese abordaje transdisciplinario plantea problemas filosóficos, sociales y culturales complejos. Al respecto, me voy a centrar exclusivamente en algunas de las dificultades metodológicas que surgen en la confluencia de tradiciones de investigación diferentes: las de las ciencias naturales con las de las ciencias sociales.

Redes digitales, comunicación social y ciencias duras Voy a partir de tres observaciones empíricas. La primera es bastante obvia: las redes digitales juegan un papel cada vez más importante en el escenario de las comunicaciones humanas. El uso de Internet y la telefonía móvil está integrado a las actividades que diariamente llevan a cabo una enorme cantidad de personas, e incide de manera significativa en el modo en que ellas 
A partir de la publicación de esos modelos, físicos, matemáticos e informáticos se abocaron a estudiar los fenómenos sociales que se dan en las redes digitales.

Como consecuencia, se produjo una explosión de investigaciones de buena calidad que hacen un uso intensivo de métodos matemáticos y estadísticos, normalmente empleados en las ciencias duras, para el estudio de fenómenos sociales. El ambiente favorable a esta explosión está dado por ese diluvio de datos procesables a que hice referencia.

Nos encontramos entonces frente a un proceso que tiene como producto a la TR, lo que supone un endurecimiento de las ciencias sociales $\mathrm{y}$, dentro de estas, de la investigación de los fenómenos de comunicación humana en las redes digitales.

La matemática de las redes complejas:

presentación de algunos resultados

El objeto de estudio de la TR son las redes complejas, lo que la inscribe dentro de una disciplina más amplia: la teoría (o ciencia) de la complejidad, cuyo objeto es el estudio de los sistemas complejos.

El concepto de complejidad, para la ciencia que se ocupa de ella, no coincide con el que utilizamos en el lenguaje corriente. ${ }^{6}$ Si bien los teóricos de la complejidad no están totalmente de acuerdo en cuanto a las características que definen a un sistema complejo, me quedaré con las que propone Mitchell (2009, pp. 12-13).

La primera es la que aquí más interesa: todo sistema complejo está compuesto por un gran número de entidades individuales que interactúan de alguna manera, formando una red. De estas redes se ocupa la TR.

Cuando analizamos un sistema complejo centrándonos en la red que contiene, como es el caso de la Web, no nos fijamos en el contenido de sus páginas, sino únicamente en cómo estas se relacionan entre sí a través de hipervínculos. Podemos considerar a la red formada por el entramado de hipervínculos que relacionan a las páginas como el esqueleto de ese sistema complejo que es la Web.
Las restantes características de los sistemas complejos son, muy brevemente:

\section{- Ausencia de un control centralizado.}

- Funciones simples cumplidas por las entidades en forma individual generan a nivel macroscópico un comportamiento colectivo que tiene propiedades emergentes para nada simples.

- Procesan información tanto internamente como con el ambiente en que se encuentran insertos, y tienen capacidad de autoorganización.

- Muchos sistemas complejos son adaptativos (tienen la capacidad de coevolucionar con el entorno en el que se encuentran).

Son ejemplos de sistemas complejos, además de la Web, la economía de un país, su sistema financiero, los ecosistemas, nuestro cerebro, Facebook, Twitter, etcétera.

En términos generales, las redes digitales de que nos servimos para comunicarnos son redes complejas. La enorme cantidad de entidades que las componen, y lo intrincado de las relaciones que las vinculan, hacen que sea imposible estudiarlas en profundidad a nivel macroscópico sin recurrir a un arsenal matemático y estadístico sofisticado. La matemática de las redes complejas apunta básicamente a atacar tres tipos de problemas:

1. Entender la topología de las redes, haciendo un análisis estático. Para esto se apoya en la teoría de grafos. Sin valernos de ella poco es lo que podemos avanzar en la comprensión de la estructura de las redes de alta complejidad como las que funcionan en Internet.

2. Entender los fenómenos de dinámica, analizando, por un lado, cómo circula la información dentro de una red y, por otro, cómo evoluciona la red. Para esto se emplea un importante abanico de técnicas estadísticas (entre ellas ocupan un lugar destacado las de la mecánica estadística). 
3. Entender las relaciones entre topología y dinámica, o sea, cómo la estructura de una red afecta su dinámica, y recíprocamente. Es en este aspecto donde aparecen los problemas más complicados.

Acabo de hacer referencia a algunos conceptos -como topología de redes, teoría de grafos o mecánica estadistica- que quizá no resulten conocidos a buena parte de los lectores, por lo que voy a intentar aclararlos brevemente.

Toda red -por ejemplo, la Web o las relaciones de amistad en Facebook - se puede representar mediante un grafo. Este es una entidad matemática que tiene básicamente dos tipos de elementos: nodos y aristas.

Los nodos se representan gráficamente como pequeños círculos, y las aristas como líneas tendidas entre ellos. Una arista tendida entre dos nodos nos dice que hay una relación entre ellos. Si observamos los grafos que representan a Facebook y a la Web, advertiremos que en los dos casos hay círculos (nodos) y líneas (aristas), tanto para los usuarios de Facebook y sus relaciones de amistad como para las páginas Web y sus hipervínculos. ${ }^{7}$

Cuando pasamos de la red al grafo que la representa, dejamos de lado las particularidades de los nodos y la naturaleza de las relaciones entre ellos. Lo que nos interesa es la topología de la red, que queda definida por la estructura de las relaciones entre las entidades que componen esa red. Al representarla mediante un grafo, obtenemos lo que puede llamarse el esqueleto matemático de la red (Plottier, 2008). ${ }^{8}$ Estamos entonces en condiciones de analizar la topología de la red mediante los instrumentos matemáticos de la teoría de grafos.

Pero no alcanza con hacer un análisis estático de las redes. También interesan los fenómenos de dinámica, que involucran la interacción de un enorme número de nodos. Ya hemos visto que, en el plano de las abstracciones matemáticas, no importa si esos nodos son páginas Web o personas en Facebook. Lo que importa es que son muchísimos e interactúan, generando esas propiedades emergentes a las que aludimos al hablar de las características de los sistemas complejos.

Es por este lado que las técnicas de la mecánica estadística tienen utilidad, ya que se trata de una disciplina que se ocupa de estudiar, en términos probabilísticos, cómo interactúa una enorme cantidad de entidades individuales, lo que da lugar a la emergencia de propiedades a nivel macroscópico (como la temperatura de un gas, resultante de la interacción de la enorme cantidad de moléculas que lo componen, para tomar un ejemplo clásico).

Los seres humanos somos entidades mucho más complicadas que los átomos o las moléculas, y nuestro comportamiento individual puede ser imprevisible y errático, pero cuando se analizan a nivel agregado los resultados de las interacciones entre un gran número de individuos aparecen regularidades estadísticas. En muchos casos -y no pocos fenómenos de comunicación caen en esta categoría-, esas regularidades que emergen a nivel macroscópico no dependen de las particularidades de las entidades a nivel microscópico (nosotros, por ejemplo, con nuestra carga personal de peculiaridades). En situaciones como estas la mecánica estadística es una importante fuente de instrumentos para el estudio de esas propiedades globales emergentes (Castellano, Fortunato, y Loreto, 2009).

Seguidamente presentaré de manera muy sumaria algunos de los resultados obtenidos por la TR, comenzando por tres conceptos básicos referentes a la topología de las redes, puestos de manifiesto inicialmente por los modelos de Watts y Strogatz, y de Barabási y Albert: el fenómeno de los mundos pequeños, el coeficiente de clusterización, y la distribución estadística del grado de los nodos.

Prácticamente todas las redes de comunicación que nos puede interesar estudiar se caracterizan por tener una propiedad topológica en común: presentan el fenómeno de los mundos pequeños, lo que indica que su diámetro es pequeño. ${ }^{9}$ Simplificando, esto significa que, a pesar de tratarse de redes con número de entidades muy grande, la distancia promedio entre dos nodos
7::

En rigor, como la Web se representa mediante un grafo orientado (los hipervínculos son como caminos con un sentido único), en lugar de líneas se utilizan flechas (arcos en la terminologia de la teoria de grafos), pero esa distinción no es relevante en este momento. 8::

Esta metáfora resulta más evidente cuando representamos una red no mediante un dibujo con nodos y aristas, sino en forma puramente numérica, utilizando una matriz de adyacencias. 9::

También denominado longitud del camino promedio o longitud del camino característico. Este concepto permite formular de manera precisa la popular idea de los seis grados de separación. 
es relativamente corta. Veamos un par de ejemplos para aclarar este concepto. ${ }^{10}$

Leskovec y Horvitz (2007) investigaron la red de usuarios del Messenger de Microsoft. Construyeron a partir de ella un grafo con 180 millones de nodos y 1.300 millones de aristas y determinaron, entre otras cosas, que su diámetro era 6,6, por lo que se trata de un mundo pequeño.

A su vez, Backstrom, Boldi, Rosa, Ugander y Vigna (2011), trabajando sobre Facebook, manejaron un grafo de alrededor de 721 millones de nodos y 69.000 millones de aristas (relaciones de amistad) y estimaron el diámetro en 4,74, por lo que también nos encontramos frente a un mundo pequeño.

Estos dos trabajos, como otros a los que me referiré más adelante, son buenos ejemplos de algo que ya mencioné: el análisis estadístico de conjuntos masivos de datos (big data en la jerga de la informática), producto del diluvio de información en el que estamos inmersos.

El diámetro pequeño es una propiedad con consecuencias prácticas importantes en relación con los fenómenos de comunicación: como los nodos de la red están en promedio separados por caminos cortos, la circulación de información es potencialmente rápida. Es condición necesaria, aunque no suficiente, para que la información pueda difundirse con facilidad.

Además, es una propiedad emergente a nivel macroscópico: nadie de Facebook o Microsoft diseñó las cosas para que esta característica tan conveniente se diera. Considerando otro ejemplo, lo mismo vale para la Web (Albert, Yeong y Barabási, 1999). El diámetro pequeño surge de la interacción de los millones de entidades elementales (personas o páginas Web, en nuestros ejemplos) que forman parte de las redes.

Las redes complejas en general, y especialmente las redes sociales, están clusterizadas. (Este concepto está vinculado al de transitividad del Análisis de Redes Sociales.) Tomando a Facebook como ejemplo, se observa que los amigos de un usuario en alguna medida son amigos entre sí. Esa medida se obtiene calculando el coeficiente de clusterización. El modelo de Watts y Strogatz muestra, entre otras cosas, cómo en un grafo clusterizado los vínculos débiles funcionan como atajos que acortan distancias, lo que da lugar al diámetro pequeño.

A partir de este trabajo es que se identifican las primeras redes con esa propiedad que hoy sabemos es muy general: la de presentar el fenómeno de mundos pequeños. ${ }^{11}$

El tercer concepto básico a que me referí es el de grado de un nodo, que es la cantidad de aristas que inciden en él (en el caso de Facebook está determinado por la cantidad de amigos que tiene el usuario, representado por el nodo). Contrariamente a lo que podría esperarse, el grado de los nodos en redes complejas no sigue una distribución normal. Esto se puso por primera vez de manifiesto en el modelo de Barabási y Albert, que analizaron la Web y determinaron que los hipervínculos que relacionan las páginas se distribuyen aproximadamente de acuerdo a una ley de potencia (que es una de las distribuciones estadísticas de cola pesada).

Relativamente muy pocos sitios concentran una enorme cantidad de hipervínculos, mientras que la enorme mayoría tiene muy pocos. El grado promedio no es entonces significativo (como lo sería si el grado siguiera una distribución normal) o, dicho de otra manera, no es una medida de escala. De aquí que Barabási y Albert digan que la Web, que también tiene la propiedad de ser un mundo pequeño, es una red libre de escala. Y esto no es una mera curiosidad estadística, sino que tiene consecuencias prácticas importantes: si el grado de los nodos siguiera una distribución normal, los motores de búsqueda como Google no podrían funcionar como lo hacen.

El modelo de Barabási y Albert muestra otro resultado interesante: aunque la cantidad de páginas Web aumenta en forma exponencial, su diámetro crece en forma logarítmica. Esto hace que la red no pierda su característica de mundo pequeño y, desde el punto de vista práctico, siga siendo entonces navegable. Este crecimiento logarítmico del diámetro es otra propiedad muy general de las redes complejas. 


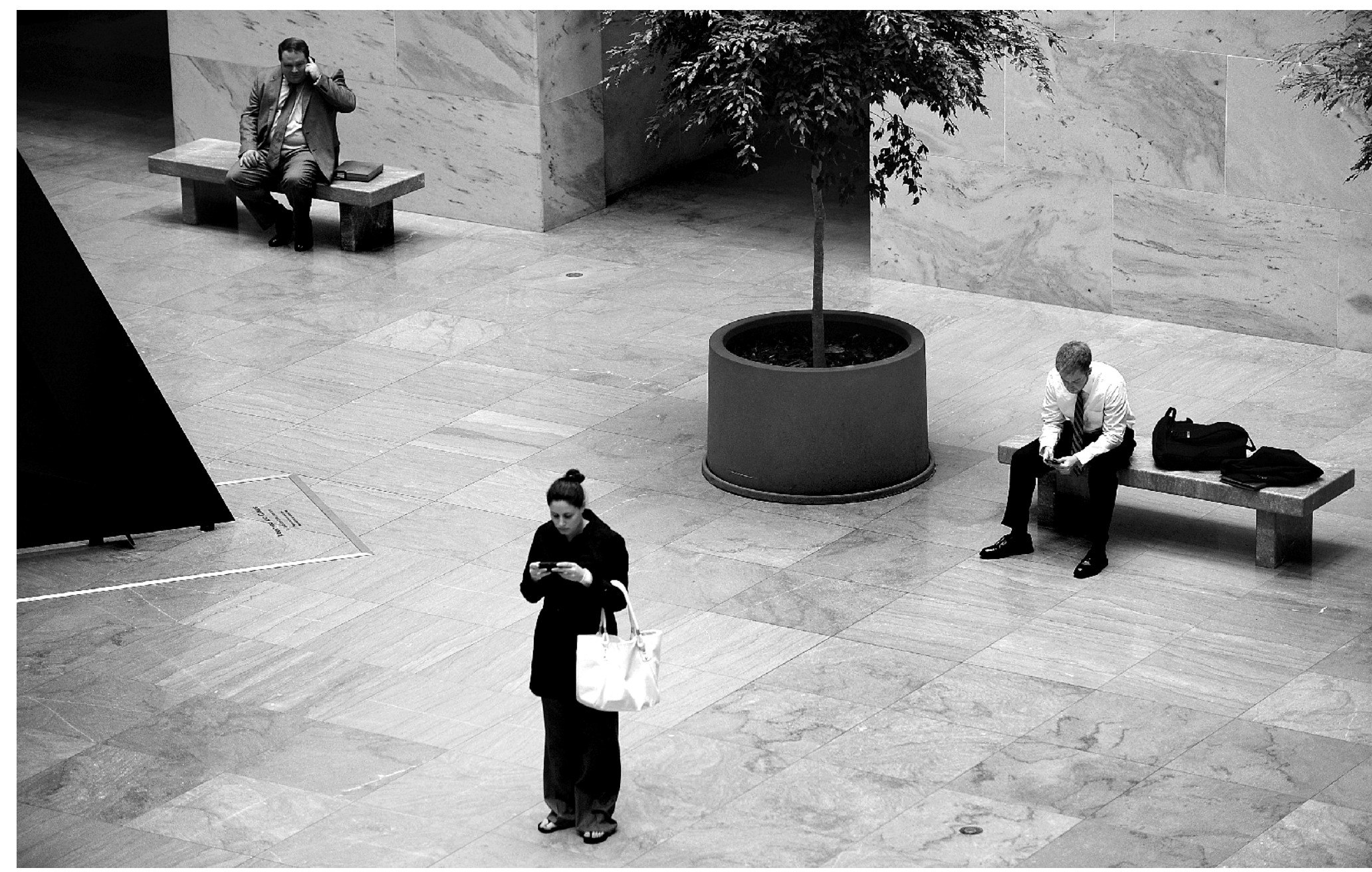

Las redes sociales complejas tienen otra propiedad importante -vinculada a los clusters-, que es la formación de comunidades. Simplificando, estas son subgrafos más densamente poblados de aristas, lo que indica que sus miembros están más relacionados entre sí que con los restantes nodos del grafo. En redes donde la cantidad de nodos y aristas es muy grande, la detección de comunidades es un problema que plantea dificultades computacionales no triviales, y es una de las áreas donde la TR está obteniendo resultados destacables (Porter, Onela, y Mucha, 2009).

Vinculado al concepto de comunidades, tenemos un problema planteado originalmente por C. Sunstein (en "Republic.com", publicado en 2001) que ha sido objeto de polémicas: Internet estaría estimulando la polarización de opiniones, produciendo así una suerte de ciberbalcanización cultural con efectos sociales negativos. Sin embargo, en Bakshy, Rosen, Marlow, y Adamic (2012) se muestra -a través de un experimento contro- lado llevado a cabo en Facebook con 253 millones de usuarios- que la información a que ellos están expuestos es más diversa de lo que podría esperarse si la polarización antes referida fuera cierta.

Aunque sobre el tema de la presunta ciberbalcanización (y problemas asociados como el de la homofilia) hay mucho por investigar, la TR, mediante un cuidadoso tratamiento estadístico de los datos, aporta evidencia empírica que contribuye a un enriquecimiento del análisis. Experimentos controlados que involucran a millones de usuarios, como el que se acaba de referir, son prácticamente imposibles fuera de un ambiente digital como es Internet. Empresas como Facebook, Google, Yahoo, Amazon y otras los llevan a cabo en forma casi permanente, como medio para mejorar sus servicios, y buena parte de nosotros participamos en ellos sin saberlo. En alguna medida todos nos hemos vuelto conejillos de indias digitales. 
La metodología más comúnmente utilizada es la del A/B Testing. En estos experimentos digitales están participando también empresas cuyo negocio principal no se hace a través de Internet, como las citadas más arriba. En Lewis y Reiley (2011) se da cuenta de un experimento digital con la participación conjunta de Yahoo y una empresa minorista, con el objetivo de medir los efectos de la publicidad hecha en los sitios de aquella sobre las ventas de esta. Participaron algo más de 1,5 millones de clientes y se pudo cuantificar de manera precisa el retorno de la inversión en publicidad hecha en Yahoo por el minorista. Adicionalmente, la empresa minorista recogió, gracias al experimento, información valiosísima sobre el comportamiento de sus clientes, imposible de obtener por otra vía.

Tanto Internet como la telefonía móvil presentan escenarios para la optimización de las inversiones en publicidad que no son posibles en los medios masivos tradicionales. Parece que por el camino de la experimentación digital quedará finalmente resuelto el famoso problema planteado por J. Wanamaker hace más de cien años, cuando dijo que la mitad de lo que gastaba en publicidad era un despilfarro, pero que ignoraba cuál era la mitad perdida.

Los experimentos digitales permiten atacar otros problemas, además de los mencionados más arriba. Uno de ellos, bien importante, es el de la predicción del éxito de los productos culturales. Es conocido que los ingresos de la industria del entretenimiento dependen básicamente de pocos grandes éxitos. Más precisamente, la distribución estadística que siguen los ingresos generados por los productos culturales no se ajusta a una curva normal, sino a una distribución de cola pesada. Por consiguiente, es fundamental tener la capacidad de predecir los éxitos.

Pero la historia muestra (con casos como Elvis Presley, Los Beatles, Harry Potter, Star Wars o American Idol) que la industria del entretenimiento, en la que no faltan expertos muy bien pagados que se dedican a evaluar las posibilidades de éxito de sus productos culturales, tiene muchos más errores que aciertos en este aspecto crucial de su gestión.
Watts (2011, pp. 76-81), mediante experimentos digitales en los que participaron unas 14.000 personas eligiendo piezas musicales en un ambiente con ocho mundos virtuales diferentes (MusicLab), prueba que el éxito de un producto cultural es inherentemente impredecible, debido básicamente a los efectos de la influencia social que se ejerce entre las personas. Una parte del éxito, la menor, depende de la calidad intrínseca del producto, pero la mayor parte es función de cómo las decisiones de unas personas influyen en las otras, y esto es algo muy difícil de cuantificar. La influencia social, según Watts, es entonces la causa de dos de las características de los mercados culturales: la impredecibilidad de los éxitos y la gran desigualdad de los ingresos que generan los productos que en ellos se comercializan. Resultados como este van contra ideas tradicionalmente aceptadas en la industria, por lo que generan no pocas polémicas.

Los fenómenos de influencia social son sumamente complejos, y la TR nos muestra que algunas ideas generalmente aceptadas respecto a las personas influyentes (o líderes de opinión), en las que se basan en general las estrategias de marketing viral, suponen una incorrecta simplificación de la realidad. Esto es de suma importancia, porque el marketing viral digital (o electrónico) despierta un enorme interés debido a que el boca a boca tiene potencialmente una gran capacidad de propagación en las redes digitales.

Las campañas de propagación boca a boca se basan tradicionalmente en la supuesta existencia de una cantidad relativamente pequeña de personas con características especiales: los líderes de opinión. Ellos tendrían la capacidad de influir sobre una gran cantidad de personas, y sus opiniones se propagarían a través de un contagio de naturaleza epidémica (de aquí la idea de viralidad). Una campaña de marketing viral debe apuntar entonces a convencer a los líderes de opinión, para desencadenar así una propagación boca a boca exitosa.

Pero la TR, a través del análisis estadístico de fenómenos de propagación en Internet, modelos matemáticos de simulación y experimentos digitales, muestra que 
las cosas son bastante más complicadas de lo que tradicionalmente se ha creído. Contrariamente a lo que se piensa, la difusión de ideas no es como la propagación epidémica de enfermedades: las personas influyentes obviamente existen, pero su papel en los fenómenos de propagación boca a boca es menor de lo que se creía; la predicción aceptablemente precisa de los resultados de las campañas es sumamente difícil (si no imposible), y las mediciones de influencia basadas en los métodos tradicionales son poco confiables (Watts, 2011, cap. 4).

Si bien la TR ha llevado a cabo una saludable labor de higiene conceptual al mostrar la debilidad de algunos preceptos tradicionalmente aceptados, no se ha limitado a ello. También ha logrado avances importantes en el conocimiento de fenómenos de dinámica en el contexto de la Web 2.0, como, por ejemplo, los de difusión de información, ejercicio de influencias y formación de opiniones, popularidad de contenidos y análisis de comunidades -véanse, por ejemplo, Asur y Huberman, 2010; Bakshy, et al., 2012; Bakshy, Hofman, Mason, y Watts, 2011; Cha, Haddadi, Benevenuto, y Gumandi (2010); Gomez-Rodriguez, Leskovec, y Krause (2010); Porter, Onnela, y Mucha (2009); Szabo, y Huberman, 2010; Watts, y Dodds, 2007; Wu, et al., 2011; Yang, y Counts (2010)-.

El telescopio, Internet y las ciencias sociales

Es común entre los proponentes de la TR afirmar que el diluvio de datos procesables derivados de la difusión de Internet tendrá para las ciencias sociales un efecto similar al que tuvo el telescopio para la astronomía. Estoy básicamente de acuerdo con esta afirmación, pero en el contexto de las reflexiones que hago en esta sección.

En primer lugar, un examen de los resultados producidos por la TR en los últimos años debe reconocer una significativa producción de conocimientos relevantes sobre la estructura y el funcionamiento de las redes digitales de comunicación. Esto vale sin perder de vista que son muchas más las preguntas que quedan abiertas que las respuestas formuladas.

En segundo lugar, en general esa producción de conocimientos se ha desarrollado sobre una base empírica razonablemente sólida, con mediciones cuidadosas, utilizando instrumentos matemáticos y estadísticos sofisticados, recurriendo a experimentos rigurosamente controlados cuando es posible, intentando con algún éxito formular leyes generales, elaborando modelos matemáticos fructíferos, logrando cierta eficacia predictiva y probando en no pocos casos lo erróneo de aseveraciones derivadas de la sabiduría convencional. Por último, los conocimientos producidos son pasibles de un análisis crítico y una eventual refutación mediante el mismo abordaje metodológico que los generó.

En tercer lugar, se puede inferir de lo anterior que la TR ha inyectado una saludable dosis de endurecimiento en el ámbito de las ciencias sociales y, dentro de estas, en el estudio de los fenómenos de comunicación. Como enfoque transdisciplinario, parece haber sorteado con éxito la frontera entre las ciencias naturales y las sociales para contribuir al avance de estas con los métodos de aquellas.

Si bien creo que lo anterior es básicamente cierto, el proceso seguido y el estado actual del arte merecen algunas consideraciones asociadas al carácter transdisciplinario de la TR. Esta se desarrolla en el contexto problemático que naturalmente se da cuando se traspasan las fronteras de las disciplinas académicas. La progresiva especialización de la ciencia a partir del siglo XVII tiene una ventaja epistemológica: ha permitido una indudable profundización del conocimiento. Pero asociada a esa especialización se da una tendencia a la compartimentación de las disciplinas, con fronteras intelectuales difíciles de sortear. Esto conspira contra el avance de la ciencia, al dificultar la fertilización cruzada de conocimientos cuando ella es necesaria.

Que diferentes tribus académicas trabajen en forma integrada atacando problemas que están en la confluencia de sus disciplinas no es entonces cosa fácil. ${ }^{12}$ La TR no es ajena a estas dificultades, que se reflejan en el plano metodológico. Agrupo estas dificultades en dos clases: en la primera incluyo problemas metodológicos concretos presentes en algunas investigaciones,
$12::$

Esto no ha impedido que existan éxitos destacados. Algunos de ellos han dado lugar a la formación de nuevas disciplinas, como la bioinformática, para tomar un ejemplo reciente. 
que se resuelven dentro del marco actual de la TR, y en la segunda un problema más general, que está en la base de los anteriores, pero dada la naturaleza de los fenómenos de comunicación presenta obstáculos más difíciles de sortear.

Los problemas metodológicos de la primera clase pueden a su vez agruparse básicamente en tres categorías: los relativos a la selección e interpretación de los datos empíricos que se toman en cuenta en los modelos matemáticos, los vinculados a la generalidad de las leyes que se postulan, y los relacionados con la capacidad explicativa y predictiva de los modelos. Voy a examinar ejemplos de estos problemas, verificados en tres dominios cognitivos diferentes.

Con respecto a la primera categoría, hay que tener presente que modelizar es simplificar. Elaborar un modelo implica seleccionar e interpretar debidamente los datos de la realidad que son relevantes con relación al problema a estudiar, tarea que no es para nada trivial en el caso de fenómenos complejos. Si ese proceso de selección e interpretación no se cumple de manera correcta, el modelo arrojará resultados inválidos, más allá de lo sofisticado del instrumental matemático que se utilice.

Un ejemplo concreto de este problema lo tenemos en las afirmaciones de Barabási sobre lo que él denominó “el talón de Aquiles" de Internet (Barabási, 2002, pp. 109-122): esta sería vulnerable a ataques debido a que posee una topología libre de escala. Pero ocurre que esa debilidad no es tal, como argumentan Willinger, Alderson y Doyle (2009), debido a que las afirmaciones relativas al supuesto talón de Aquiles de Internet se basaron en datos empíricos incorrectos. ${ }^{13}$

Este ejemplo nos muestra dos facetas, una negativa y otra positiva. La negativa tiene que ver con el riesgo que se origina cuando científicos de una disciplina (los físicos, por ejemplo), encandilados por la abundancia de datos empíricos, aplican sus métodos y esquemas mentales a un dominio que les es ajeno (la ingeniería de Internet, en nuestro ejemplo), sin preocuparse por conocer en profundidad las particularidades del campo del conocimiento en el que están incursionando. Esto puede llevarlos a recolectar datos que no son los pertinentes, utilizar mediciones que no son las apropiadas y llegar a conclusiones erróneas, como ocurrió en el ejemplo considerado.

La faceta positiva tiene que ver con la refutabilidad de las aseveraciones hechas en el marco de la TR. Willinger, et al. (2009), examinando el mismo universo de información empírica utilizado para sustentar la afirmación sobre el talón de Aquiles de Internet, pero seleccionando los datos correctos y haciendo las mediciones apropiadas, probaron que dicha afirmación es incorrecta. Los resultados de la TR están expuestos a la contrastación empírica y son refutables, algo muy saludable para el desarrollo del conocimiento científico.

Como segunda categoría de problemas, se observa una tendencia a otorgar excesiva generalidad e importancia a ciertas regularidades empíricas. Esto no debería extrañar, ya que los físicos - hasta ahora mayoría entre los investigadores en TR- provienen de una larga y exitosa tradición de investigación que privilegia la búsqueda de leyes universales, y es natural que mantengan esta orientación al aplicar sus conocimientos a otros dominios de la realidad.

Un ejemplo de esta actitud lo tenemos en Barabási y Bonabeau (2003), donde de la ubicuidad de las redes libres de escala se infiere la existencia de una arquitectura general de los sistemas complejos regulada por leyes fundamentales, que los autores consideran aplicables a las células, las computadoras, las lenguas y las sociedades. Afirmaciones tan ambiciosas como esta no están de acuerdo con la realidad imperante en los dominios del conocimiento aludidos.

Veamos brevemente un caso destacado: el de redes biológicas. Como observa Keller (2005), algunos investigadores de la TR atribuyen a las redes libres de escala y a las leyes de potencias una generalidad y una significación excesivas, no respaldadas por un examen cuidadoso de los datos empíricos de la biología. No hay evidencia que permita afirmar, como se ha hecho, que las redes libres de escala prueben que existe una arqui- 


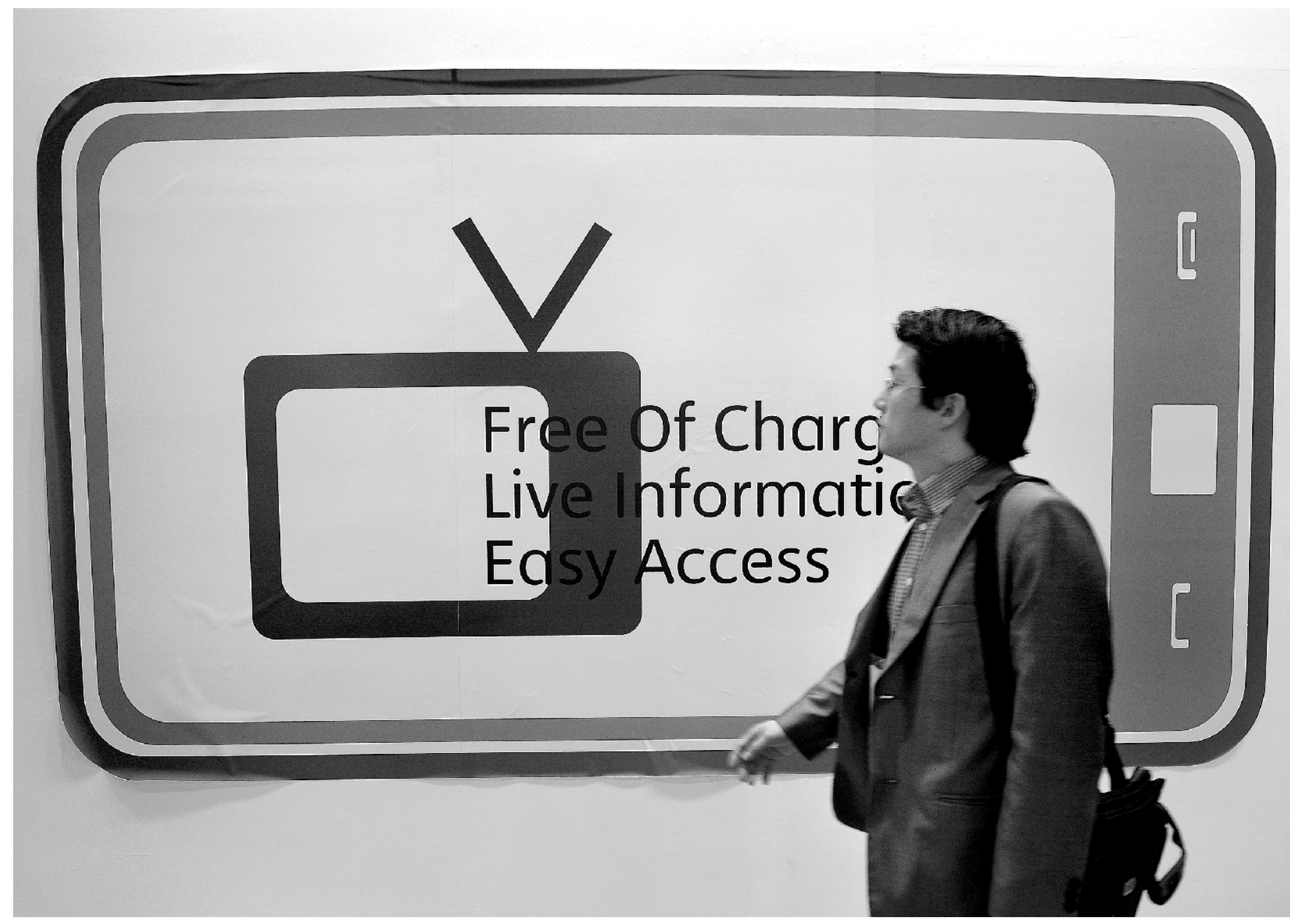

tectura general de los sistemas biológicos, o que constituyen la expresión matemática de una de las leyes fundamentales de la vida. Generalizaciones del alcance que es habitual en el campo de la física parecen no ser posibles, según los biólogos como Keller, en el dominio de las ciencias biológicas. Como veremos seguidamente, vale lo mismo para las ciencias sociales.

Entrando en la tercera categoría de problemas, ciertos resultados propuestos por algunos investigadores en TR respecto a la capacidad predictiva y explicativa de algunos modelos lucen exageradamente optimistas. En el aspecto predictivo, este optimismo está asociado a la recién comentada fe excesiva en la generalidad de ciertas leyes, complementada por cierto apresuramiento en encontrar mecanismos explicativos mediante el uso de modelos matemáticos.
Barabási (2010, pp. 10-11) afirma que la mayoría de nuestras acciones están reguladas por leyes de amplio alcance, las que reflejan un orden profundo en el comportamiento humano, y cuyo poder predictivo es comparable al que encontramos en las ciencias naturales. En el ámbito de las ciencias sociales no parece justificarse este optimismo, tanto en lo que hace a la existencia de leyes generales como a la capacidad predictiva de la TR.

D. Watts (2011, pp. 141-143, 261) sostiene que buena parte de los fenómenos sociales presentan dificultades predictivas prácticamente insalvables (hemos visto un par de ejemplos en la sección anterior). La raíz de esta dificultad está en que un componente esencial de los fenómenos sociales es la interacción entre las personas, la que genera un juego de influencias que se
Foto: Jung Yeon-Je, AFP. 
retroalimentan en forma no lineal y dan lugar a escenarios muy complejos, donde las variables que se intenta medir evolucionan muchas veces de forma no previsible. Paradójicamente, las ciencias blandas presentan problemas más duros que las ciencias duras.

Hacer predicciones en relación con fenómenos sociales es posible, y la TR muestra resultados destacables en este sentido, pero no hay evidencia que justifique el optimismo fisicalista de algunos investigadores. ${ }^{14}$

Adentrarse en el territorio de los fenómenos sociales ofrece entonces dificultades que no presenta el de las ciencias naturales. Y esto nos lleva a analizar la segunda clase de problemas metodológicos: los asociados a la naturaleza de los fenómenos de comunicación. Estos presentan sin duda dimensiones duras y blandas, y solo los comprenderemos integralmente si articulamos debidamente las dos dimensiones.

Del lado de las ciencias sociales tenemos una rica tradición de investigación cualitativa que se ocupa de las dimensiones blandas, así como también el uso de métodos duros, cuantitativos, aunque con enfoques diferentes al de la TR. Esta, por su parte, consistentemente con la tradición de investigación de la que proviene, se centra en las dimensiones duras a nivel macroscópico. ${ }^{15}$

Ocurre que entre ambas tradiciones de investigación hay distancias aún no bien salvadas. Anoto que esto mismo está en la base de los casos antes señalados Con relación a la ingeniería de Internet y la biología, pero los problemas se incrementan cuando se necesitan puntos de contacto entre ciencias duras y ciencias sociales.

El problema central está entonces en lograr una convergencia metodológica entre ambas tradiciones de investigación, tendiendo mayor cantidad de puentes entre los abordajes duros y blandos de los fenómenos de comunicación, sin que el desarrollo de unos vaya en menoscabo de otros. Esto es una necesidad ineludible dada la doble dimensión de los fenómenos de comunicación social.
No se me escapa una objeción que puede hacerse a esta propuesta de convergencia metodológica: los seres humanos seríamos demasiado complejos como para que los métodos de las ciencias naturales tengan éxito con los problemas sociales. De acuerdo con esto, la metáfora de Internet como telescopio, aludida al principio de esta sección, sería exagerada.

Sin entrar en las consideraciones filosóficas que el punto merece, pienso que los resultados obtenidos hasta ahora por la TR abonan en contra de esta objeción. Pero si los puentes entre las disciplinas no se tienden, acercando los respectivos territorios, la metáfora del telescopio será una mera expresión de deseos, y el avance del conocimiento científico en materia de comunicación no tendrá la riqueza que el tema merece.

Ahora bien, ¿es posible mirar con optimismo ese tendido de puentes intelectuales, acercando los diferentes abordajes? Mi conjetura es que sí, sin por esto dejar de reconocer las dificultades que implica hacer entrar en un diálogo estrecho a comunidades académicas con culturas muy diferentes.

Mi optimismo se apoya en primer lugar en lo que está ocurriendo en los laboratorios de investigación de empresas como Yahoo, Microsoft, Intel, Google y Nokia, para mencionar algunas. Llevan a cabo investigaciones, y en no pocos casos publican trabajos científicos, mediante equipos multidisciplinarios en los que participan, por ejemplo, informáticos, matemáticos, físicos, sociólogos, antropólogos y economistas. Esto prueba que la convergencia metodológica no es una utopía.

En segundo lugar, un examen de la literatura científica producida por la TR permite observar una tendencia creciente a citar autores del dominio de las ciencias sociales, como Lasswell, Katz, Lazarsfeld, Merton, Granovetter, Milgram, Coleman y otros. Parece que los científicos duros están haciendo los deberes, estudiando la producción académica de las ciencias sociales. Si bien estamos en presencia de un comienzo, no es poca cosa.

Mi optimismo se ve algo atenuado porque no percibo del lado de las investigaciones en comunicación una 
preocupación suficiente por interiorzarse en los avances de la TR. Dado que las redes digitales tienen una importancia creciente en nuestra vida diaria, y que los fenómenos de comunicación se caracterizan por tener dimensiones duras y blandas, esta situación debería modificarse.

En conclusión, si bien me parece factible que las ciencias sociales den con Internet un salto epistemológico comparable al de la astronomía con el telescopio (sin desconocer las enormes diferencias entre ambos dominios del conocimiento), todavía queda un largo y difícil camino por recorrer, con problemas que no deben subestimarse. Para que la metáfora del telescopio sea una realidad, será necesario intensificar el tendido de puentes a que aludí más arriba, de manera que la investigación en comunicación pueda dar el salto epistemológico que la realidad del siglo XXI requiere. :-

\section{Referencias}

Albert, R., H. Jeong, y A. L. Barabási (1999). Diameter of the World Wide-Web. Recuperado de http://www.barabasilab. com/pubs/CCNR-ALB_Publications/199909-09

Asur, S., y B. Huberman (2010). Predicting the Future With Social Media. Recuperado de: http://www.hpl.hp.com/ research/scl/papers/socialmedia

Backstrom, L., P. Boldi, M. Rosa, J. Ugander, y S. Vigna (2012). Four Degrees of Separation. Recuperado de http:// arxiv.org/abs/1111.4570

Bakshy, E., J. Hofman, W. Mason, y D. Watts (2011). Everyone's an Influencer: Quantifying Influence on Twitter. Recuperado de research.yahoo.com/files/wsdm333w-bakshy.pdf

Bakshy, E., I. Rosenn, C. Marlow, y L. Adamic (2012). The Role of Social Networks in Information Diffusion. Recuperado de http://arxiv.org/abs/1201.4145

Barabási, A.-L. (2002). Linked. Cambridge, Perseus Publishing. (2010). Bursts. Nueva York, Dutton.

Barabási, A.-L., y E. Bonabeau (2003). Scale-Free Networks. Recuperado de http://icosystem.com/site/wpcontent/uploads/SciAm2003.pdf

Castellano, C., S. Fortunato, y V. Loreto (2009). Statistical physics of social dynamics. Recuperado de http:// arxiv.org/abs/0710.3256

Cha, M., H. Haddadi, F. Benevenuto, y K. Gummadi (2010).
Measuring User Influence in Twitter: The Million Follower Fallacy. Recuperado de an.kaist.ac.kr/ mycha/docs/ icwsm2010_cha.pdf

Gomez-Rodriguez, M., J. Leskovec, y A. Krause (2010). Inferring Networks of Diffusion and Influence. Recuperado de http://cs.stanford.edu/people/ jure/pubs/netinf-kdd2010

Hilbert, M., y P. López (2012). The World's Technological Capacity to Store, Communicate, and Compute Information. Recuperado de http://www.sciencemag.org/ content/332/6025/60

Keller, E. F. (2005). Revisiting "Scale-free" networks. Recuperado de filer.case.edu/users/ngb4/Fox_Keller_ Article.pdf

Leskovec, J., A. Singh, y J. Kleinberg (2006). Patterns of Influence in a Recommendation Network. Recuperado de www.cs.cornell.edu/home/kleinber/pakdd06-cascade.pdf

Leskovec, J., y E. Horvitz (2007). Planetary-Scale Views on an Instant-Messaging Network. Recuperado de http: http://arxiv.org/pdf/0803.0939v1.pdf

Lewis, R., y D. Reiley, D. (2011). Does Retail Advertising Work? Recuperado de http://www.davidreiley.com/papers/ DoesRetailAdvertisingWork.html

Mitchell, M. (2009). Complexity. Nueva York, Oxford University Press.

Plottier, J. L. (2008). Medios de comunicación y teoría de redes. Montevideo, Universidad Católica del Uruguay.

Porter, M., J.-P. Onnela, y P. Mucha (2009). Communities in Networks. Recuperado de http://arxiv.org/abs/0902.3788

Szabo, G., y B. Huberman (2010). Predicting the Popularity of Online Content. Recuperado de http://arxiv.org/pdf/ 0811.0405v1.pdf

Watts, D. (2011). Everything is obvious. Nueva York, Crown.

Watts, D., y S. Dodds (2007). Influentials, Networks, and Public Opinion Formation. Recuperado de research.yahoo.com/ files/w_d_JCR.pdf

Willinger, W., D. Alderson, y J. Dyle (2009). Mathematics and the Internet: a Source of Enormous Confusion and Great Potential. Recuperado de http://www.ams.org/notices/ 200905/tx090500586p.pdf

Wu, S., J. Hofman, W. Mason, y D. Watts (2011). Who Says What to Whom on Twitter. Recuperado de research.yahoo.com/ files/twitter-flow.pdf

Yang, J., y S. Counts (2010). Predicting the Speed, Scale, and Range of Information Diffusion in Twitter. Recuperado de www.aaai.org/ocs/index.php/ICWSM/ICWSM10/ paper/view/1468/1896 